\title{
SOBRE LA FORMACIÓN DE GRUPOS CLÍTICOS ROMÁNICOS EN EL COMPONENTE POSTSINTÁCTICO ${ }^{1}$
}

\author{
$M^{a}$ Victoria Camacho Taboada \\ Universidad de Sevilla
}

\begin{abstract}
Surprisingly, Romance and Slavic clitic clusters show similar morphological and morphonological behaviours despite their different syntactic status as $\mathrm{D}^{\min / \max }$ or $\mathrm{Agr}^{\min / \max }$. This means clusters made by both clitic types should be created in the same linguistic level. In particular, we argue their internal order may be controlled by a set of ranked, violable morphonological constraints applied in a postsyntactic component as in Optimality Theory.
\end{abstract}

\section{INTRODUCCIÓN.}

Uno de los fenómenos más interesantes relacionados con la distribución sintáctica de los clíticos en general consiste en que si dos o más clíticos de la misma clase coinciden en una misma cláusula, éstos se aglutinan formando una secuencia estrictamente ordenada. ${ }^{2}$ Algunos autores consideran que esta combinación se produce en el componente sintáctico asumiendo, por un lado, que la estructura interna del grupo clítico es jerárquica y, por otro, que su disposición en contextos de enclisis y proclisis es especular. Sin embargo, los datos parecen mostrar todo lo contrario. Estas amalgamas se caracterizan precisamente por no seguir una estructura jerárquica al tiempo que su orden interno es el mismo en todos los contextos; esto es, tanto en cláusulas simples o complejas con independencia de su estatuto sintáctico, de su distribución enclítica o proclítica o de si están implicados en construcciones de subida o doblado. Además, se trata de combinaciones no productivas como demuestra el hecho de que no están permitidas todas las secuencias lógicamente posibles o el hecho de que la combinación de dos de ellos con similitudes fónicas provoque la aparición de formas especiales (que no se producirían en el caso de que aparecieran solos en la cláusula). Todos estos datos apuntan a que estamos tratando con una unidad morfológica y no sintáctica. Concretamente, en este artículo argumentaremos que los grupos clíticos se forman en un nivel morfológico postsintáctico.

Analizaremos, en primer lugar, las características más relevantes asociadas con la formación de los grupos clíticos, a saber, el orden interno al que deben ajustarse y los fenómenos de alomorfía que suelen producirse en dichas secuencias. Señalaremos que las

\footnotetext{
${ }^{1}$ Este artículo está dedicado a la memoria del Profesor Feliciano Delgado León.

${ }^{2}$ Dichas secuencias están formadas por clíticos que realizan diferentes funciones sintácticas. Como consecuencia el nudo resultante de la formación del grupo no puede ser etiquetado con una etiqueta concreta; esto es, se trata de una unidad polifuncional.
} 
propuestas sintácticas no son adecuadas para dar cuenta de la formación de estas unidades. Para ello tomaremos como referencia el comportamiento de los grupos clíticos de las familias lingüísticas románicas y eslavas. De hecho, consideramos que se trata de un fenómeno independiente de los principios que regulan su distribución en la cláusula. En concreto, argüiremos que se forman (con independencia de su estatuto sintáctico) en el componente morfológico situado en el interfaz sintáctico-fonológico.

Además, argumentaremos que dicho interfaz es el encargado de traducir la información sintáctica proveniente del componente sintáctico en información fonológica. En este punto de la derivación, se producirían los siguientes procesos: en primer lugar, un proceso de linealización de los nudos terminales sintácticos en el que intervienen ciertos principios de la Teoría de la Optimidad; en segundo lugar, un proceso de empobrecimiento morfológico, en la línea de la Morfología Distribuida, por el que los rasgos morfosintácticos que componen los objetos sintácticos asociados a los grupos clíticos son traducidos a información exclusivamente morfológica; y por último, se produce el proceso de inserción léxica por el que dichos rasgos morfológicos son traducidos en información fonológica.

\section{DATOS Y PLANTEAMIENTO DEL PROBLEMA.}

\subsection{El orden interno del grupo clítico.}

En la mayoría de los sistemas lingüísticos románicos, los grupos clíticos están formados, además de por clíticos pronominales de objeto, por clíticos pronominales partitivos $^{3}$ y locativos $^{4} \mathrm{y}$, en el caso de los eslavos, por clíticos auxiliares e interrogativos. Esto hace que sea complicado definir mediante un conjunto reducido de criterios su orden interno. No obstante, en términos generales, podemos decir que éste parece estar condicionado, en primer lugar, por la función sintáctica que realizan y, en el caso de que intervenga más de un clítico pronominal, por el caso y/o la persona gramatical que representan.

Uno de los criterios utilizados por Richard Kayne (1975) para caracterizar el comportamiento de los estos elementos consiste en que éstos siguen un orden fijo que, a menudo, difiere del orden que, en ese mismo sistema lingüístico, siguen los sintagmas o frases en la sintaxis. Esta característica parece indicar que los clíticos tienen un

\footnotetext{
${ }^{3}$ Encontramos clíticos con función partitiva en italiano, francés, catalán, checo, esloveno y polaco:

(i) Esloveno

Jano ich pozná vela

(Vos y Veselovská 1999:918)

Jano 3pAcc-Gen knows many

Jano knows many of them

${ }^{4}$ Los clíticos locativos están presentes en italiano ( $c i$ y ne), francés ( $y$ y en) y catalán ( hi y en). Cada uno de ellos ha desarrollado dos formas de locativo pronominal del ibi e inde latinos, respectivamente. Más específicamente, Corominas (1954-7) considera que el clítico de locativo hi del catalán proviene de la confluencia del elemento demostrativo hic y el adverbial ibi (vid. Longa et al. 1998).
}

(i) Catalán

(Longa et al 1998:137)

A la portada, l'editor hi posa el nom de l'autor

On the cover the editor LOC put the name of the author

On the cover, the editor puts the name of the author 
comportamiento similar al de los afijos. ${ }^{5}$ De hecho, Perlmutter (1970) compara este fenómeno con las restricciones de orden a las que están sometidos los afijos en los sistemas lingüísticos polisintéticos. Al igual que ocurre en estos sistemas, es difícil describir la formación del complejo clítico y anfitrión como la adición progresiva y jerarquizada de clíticos a una base. Más bien, se trata de cadenas lineales de elementos adjuntadas a un tiempo a dicha base. Como consecuencia, este orden suele ser representado a modo de plantilla (vid. Perlmutter 1970, 1971; Simpson y Withgott 1986; Bonet 1993; Sportiche 1996; Monachesi 1999) 6 .

En cuanto a los grupos eslavos, existe cierta variación en el orden que ocupan los clíticos auxiliares, reflexivos e interrogativos en relación con los pronominales argumentales no reflexivos. Franks y King (2000:205) recogen los siguientes patrones a modo de plantilla:

(1) Orden del grupo clítico en los sistemas lingüísticos eslavos.

\begin{tabular}{ll}
\hline Sistema Lingüístico & Orden \\
\hline Serbocroata & Li $>$ AUX $>$ DAT $>$ ACU $>$ GEN $>s e>j e$ \\
Esloveno & $n a j>b i /$ AUX $>$ REFL $>$ DAT $>$ ACU $>$ GEN $>$ FUT AUX $/ j e$ \\
Búlgaro & ste $>$ AUX $>$ DAT $>$ ACU $>e$ \\
Macedonio & $d a>n e>k e / b i>$ AUX $>$ DAT $>$ ACU $>$ e $/$ se \\
Checo & $l i>$ COND $/$ AUX $>$ DAT - NO ARG. $>$ DAT $>$ ACU $>$ GEN \\
Eslovaco & $b y><$ AUX REFL $>$ DAT-NO ARG. $>$ DAT $>>$ ACU $>$ GEN \\
\hline
\end{tabular}

La información implementada en estos moldes sólo representa el orden lineal en que estos deben aparecer y no su combinación. Así pues, debe tenerse en cuenta que: (i) cada posición sólo puede estar ocupada por un elemento, es decir, no pueden coexistir dos clíticos del mismo grupo en una secuencia; (ii) sólo están permitidas combinaciones con un

${ }^{5}$ Esta afirmación parte de la tesis de que el orden de los constituyentes de la palabra es fijo en el sentido de que un cambio en el orden implica un cambio de significado. No obstante, se trata de un criterio orientativo. Así, en quechua el sufijo delimitativo nominal la puede ocupar opcionalmente varias posiciones en la palabra sin que se produzca ningún cambio de significado (Riemsdijk 1999:8):

(i) Quechua
a. Kiki-la-n-kuna
Self $3 \mathrm{pl}$
Just themselves
b. Kiki-n-kuna-la
c. Kiki-la-n-kuna-la

Muysken (1981:295) ${ }^{6}$ Perlmutter (1970) se basa en los estudios de los descriptivistas americanos quienes describen el comportamiento
de los afijos de estos sistemas lingüísticos como elementos que se amoldan a plantillas en las que se fijan una serie
de posiciones que han de ser saturadas por estos afijos. Asimismo, se caracterizan porque, cuando se combinan
entre sí, no es posible determinar su núcleo y porque su forma puede estar condicionada por la presencia de ciertos
afijos adyacentes. Dos características que comparten con los grupos clíticos.
La noción de plantilla ha sido formalizada en la teoría generativa como filtros que han de ser activados tras la
aplicación de las reglas de la sintaxis, si bien los autores difieren en cuanto al lugar exacto en que estos filtros
deben ser colocados. Así, Perlmutter (1970) opina que deben ser aplicados a la salida del componente sintáctico,
mientras Bonet (1993) considera que este mecanismo debe ser implementado en el componente morfológico,
situado en el interfaz sintáctico-fonológico. 
número limitado de clíticos que en el caso de los sistemas eslavos suele ser de tres y en los sistemas románicos dos.

No nos detendremos en describir con detalle estas variaciones, pero baste decir que los clíticos pronominales no reflexivos actuarían como el núcleo del grupo alrededor del cual se sitúa el resto. Siguiendo a Franks y King (2000:206), el patrón de actuación común es el siguiente: los clíticos reflexivos aparecen bien a la izquierda (2) o bien a la derecha del resto de los clíticos (3); los clíticos auxiliares preceden a los pronominales (4), excepto en algunos sistemas lingüísticos en los que los clíticos de tercera persona auxiliares aparecen en el último lugar del grupo $(5)^{7}$; y por último, el clítico interrogativo $l i$ aparece en la primera posición de la secuencia (5): ${ }^{8}$

(2) Esloveno Franks y King (2000:206)

Predstavil sem se mu

Introduce. Prt aux.1sg refl.ACU him.DAT

I introduced myself to him

(3) Macedonio

Franks y King (2000:206)

Mi se stori deka toj si odi

Me.DAT refl.ACU seemed.3sg that he refl.DAT leave.3sg

It seemed to me that he was leaving

(4) Eslovaco

Franks y King (2000:206)

Bál som sa jej

Feared aux.1sg refl.Acu her.gen

Ifeared her

(5) Serbocroata

Franks y King (2000:206)

Da li mu ga je možda Goran dao?

C Q him.DAT it.Acu Aux.3sg maybe Goran gave

Has Goran perhaps given it to him?

No obstante, si en una misma secuencia aparecen dos clíticos pronominales su orden estará determinado por su caso. Así, los argumentales siguen el orden de dativo [DAT] seguido de acusativo [ACU] (6), mientras que los de ACU preceden a los de genitivo (7).

(6) Checo

Franks y King (2000:206)

Půjčil byste mi ji na týden?

Lent cond.2pl me.DAT her/it.Acu for week

Would you lend it to me for a week?

(7) Eslovaco

Franks y King (2000: 206)

\footnotetext{
${ }^{7}$ Spencer (1991), en cambio, argumenta que en serbocroata el clítico verbal je ocupa la última posición del grupo cuando funciona como verbo copulativo, y no como auxiliar.

${ }^{8}$ El clítico interrogativo $l i$ del esloveno y checo es considerado de la clase de los clíticos simples frente al resto de los miembros del grupo que pertenecen a la clase de los clíticos especiales de acuerdo con la clasificación de Zwicky (1977).
} 


\author{
Ušetril som ju jej \\ Spared aux.1sg her.Acu it/her.gen \\ I spared her form it/her
}

Lo interesante de estos comportamientos consiste en que el patrón que siguen es el mismo para los clíticos de segunda posición del serbocroata y los clíticos categoriales del macedonio y búlgaro. En otras palabras, independientemente del estatuto sintáctico de estos elementos (esto es, si se trata de categorías $\mathrm{D}^{\text {mín/máx }}$ o Conc ${ }^{\text {mín/máx }}$ - vid. Camacho, 2002, 2004) el orden interno de estos grupos parece estar determinado por la función sintáctica y el caso que realizan en la cláusula. Esto propicia que los análisis que se han propuesto para dar cuenta de los clíticos eslavos recurran a soluciones sintácticas (vid. Progovac 1996, Franks 1998, Dimitrova-Vulchanova 1999, Franks y King 2000, entre otros).

En los sistemas lingüísticos románicos se observa que la característica que rige el orden interno del grupo es su función sintáctica. No obstante, si el grupo está formado por dos clíticos pronominales el criterio que determina su secuenciación es la persona gramatical, es decir, un rasgo morfosintáctico. Al igual que con los eslavos, observamos cierta variación interlingüística.

(8) Orden interno de los grupos clíticos románicos 9

\begin{tabular}{ll}
\hline Sistema lingüístico & Orden interno \\
\hline Español & Se II I III \\
Catalán & III \\
ACU II I III en/hi \\
Italiano & I/II/III $_{\text {DAT }} c i_{\mathrm{LOC}} s i_{\mathrm{REFL}} \mathrm{III}_{\mathrm{ACU}} s i_{\mathrm{IMP}} n e$ \\
Francés & il ne I/ II / REFL. III \\
\hline
\end{tabular}

Este sistema de manejo de información gramatical permite establecer relaciones paradigmáticas entre los clíticos, de modo que sólo se admitirá en cada secuencia uno de ellos por columna. Así, por ejemplo, en español siempre que aparezca más de uno en la misma cláusula, un clítico de segunda persona precederá al de primera y cualquiera de éstos antecederá al de tercera. El único elemento que no cumple estas reglas es se que, independientemente de su función sintáctica, siempre aparecerá en la primera posición del grupo.

No obstante, ésta no es la única restricción a la que están sometidos. Estas secuencias son, además, sensibles al caso morfológico. En el supuesto de que el grupo esté formado por dos clíticos de tercera persona, la única secuencia posible en español e italiano es DAT ACU (9). En francés, en cambio, se produce el efecto contrario; el orden permitido es ACU DAT (10).

(9) Español

a) Se lo envié a Ángela

b) *Lo se envié a Ángela

(10) Francés

\footnotetext{
${ }^{9}$ Para realizar esta comparación hemos homogeneizado el formato de las fuentes. Para ello hemos usado los datos que aparecen en Perlmutter (1970:213) para el español, Vos y Veselovská (1999:970) para el catalán, Monachesi (1999:68) para el italiano y Sportiche (1996:216) para el francés.
} 


\section{Il le lui donnera \\ Él se lo dará}

Por otro lado, no todas las combinaciones posibles están admitidas. ${ }^{10}$ Así, en romance, no están permitidos los grupos formados por clíticos de primera o segunda persona de ACU con clíticos de tercera de DAT (11a) y (11b). Esta restricción afecta exclusivamente a la formación de grupos clíticos puesto que dichas secuencias pueden ser glosadas por construcciones en las que uno de los argumentos del verbo está realizado por un pronombre tónico (11c) (vid. Perlmutter 1970). Esta restricción también afecta a los grupos del búlgaro:

(11) Italiano

Gerlach (1998:5)

a) * Emanuela mi glie presenta

b) * Emanuela glie mi presenta

c) Emanuela presenta me a lui Emanuela introduce.3sg pro.1sg P pron.3sg

Emanuela introduces me to him

(12) Búlgaro Franks y King (2000:62)
a) *Pokazvat mu me
show.3pl him.DAT me.ACC
They show me to him
b) Pokazvat mene na nego
They show me to him

Estos ejemplos incumplen las restricciones de persona (11a) y (12a), y caso (11b) y (12b). Si sustituimos el clítico de DAT de tercera persona por el clítico de ACU obtenemos una construcción gramatical:

(13) Español

$$
M e_{\mathrm{DAT}} l o_{\mathrm{ACU}} \text { recomendaron }
$$

Estas restricciones que afectan a las características léxicas de los clíticos son un indicio de que estamos tratando con unidades morfológicas. De hecho, la formación de grupos clíticos no es un fenómeno productivo y regular tal y como se espera de los fenómenos tratados desde el componente sintáctico. Por tanto, creemos conveniente que sea tratado desde el componente morfológico.

Asimismo, la combinación de un clítico pronominal de objeto de primera y segunda persona está prohibida en español, francés o italiano. En cambio, en rumano la secuencia $m i$ te sí es posible (vid. Popescu 1997):

$$
\text { (14) Italiano Gerlach (1998) }
$$

a) * Emanuela me ti presenta

\footnotetext{
10 En estas restricciones de orden no incluimos aquellas que pueden ser explicadas teniendo en cuenta las condiciones sintácticas que regulan la correferencia; por ejemplo, en español no son posibles las siguientes combinaciones (vid. Fernández Soriano 1999):
}

$$
\begin{aligned}
& \text { (i) Español } \\
& * \text { * Me nos entregó }
\end{aligned}
$$

Es decir, en un grupo clítico no pueden aparecer dos elementos con el mismo referente. 
b) * Emanuela te mi presenta

c) Emanuela presenta me a te Emanuela introduce.3sg pro.1sg P pron.2sg

Emanuela introduces me to you

(15) Italiano

Gerlach (1998)

a) * Emanuela me ti presenta me a lui

b) * Emanuela te mi presenta me a lui

c) Emanuela presenta te a me

Emanuela introduce.3sg pro.2sg P pron.1sg

Emanuela introduces you to me

De nuevo, estas construcciones pueden ser glosadas utilizando pronombres tónicos en lugar de uno de los clíticos. ${ }^{11}$

En síntesis, los análisis que se han realizado para dar cuenta del orden interno de los grupos clíticos eslavos y románicos coinciden en señalar que éstos se rigen por la función sintáctica y el caso que realizan. Y en el caso de los clíticos románicos, además, por la persona gramatical que representan. ${ }^{12}$ En este sentido, estas restricciones pueden ser interpretadas como un indicio de que los clíticos pronominales se comportan como afijos que muestran ciertos huecos arbitrarios en el paradigma tal y como es característico de las unidades morfémicas.

Asimismo, hemos visto que existe cierta variación en cuanto al orden de los clíticos inter e intralingüísticamente. Por tanto, un análisis adecuado de este fenómeno debe dar cuenta de por qué se producen estas restricciones en cuanto al caso y/o la persona

\footnotetext{
${ }^{11}$ No obstante, en español e italiano algunos hablantes consideran válida esta combinación si es interpretada como la secuencia de caso ACU DAT; una construcción que invalidaría la hipótesis de que en español e italiano debe cumplirse obligatoriamente la restricción de caso.
}

(i) Español

Te me escapaste

2Acu 1Dat escapaste

* Me te escapaste

(ii) Italiano

Gianni mi ti presentera

Riemsdijk (1999: 8)

Gianni 1Acu 2Dat presentara

* Gianni ti mi presentera

Obsérvese que la combinación sería válida si uno de los clíticos es un dativo ético:

(iii) Español

Te me vas

En cambio, para algunos hablantes del catalán dicha combinación es posible tanto con interpretación ACU DAT como con la de DAT ACU:

(vi) Catalán

(Eva Monrós, c.p.)

$T e^{\prime} m$ van presentar

Te me presentaron

${ }^{12}$ Para algunos lingüistas esta restricción parece tener validez universal no sólo en la combinación de clíticos, sino también con marcas de concordancia de objeto. Por ejemplo, un morfema de tercera persona de acusativo o absolutivo no puede coexistir con una marca de concordancia de dativo con verbos ditransitivos (vid. Bonet 1991). 
gramatical, qué mecanismo(s) o principio(s) gramatical(es) puede(n) dar cuenta de ello, y por último, cómo puede explicarse la variación interlingüística.

\subsection{Procesos morfonológicos en el grupo clítico}

Otro de los argumentos que parecen demostrar la naturaleza morfológica de los grupos clíticos, es el hecho de que en su dominio se producen ciertos procesos morfonológicos típicos del ámbito de la palabra. ${ }^{13,14}$ Bonet (1995) denomina al resultado de estos fenómenos formas opacas y las define como outputs of clitic combinations that do not coincide with the output forms of those clitics in isolation (Bonet 1995:607). En términos generales, es posible diferenciar tres clases de formas opacas de acuerdo con el tipo de modificación que sufren sus formas fónicas: en primer lugar, fusión o amalgama de los dos clíticos en una sola forma; en segundo lugar, transformación alomórfica del primer clítico del par y, por último, la supresión de uno de sus miembros. ${ }^{15}$

De acuerdo con nuestros datos, el sistema lingüístico que posiblemente muestra un mayor número de formas opacas o amalgamas es el portugués. En (16) observamos que todos los casos en los que un grupo clítico está formado por la secuencia DAT ACU obtenemos una forma contracta. Dichas formas son el resultado de la asimilación de los sonidos finales del primer miembro del grupo o de la incorporación de sonidos epentéticos:

(16) Portugués

(Cunha y Cintra 1984:309)

\begin{tabular}{|c|c|c|c|c|}
\hline & $\mathrm{DAT}+\mathrm{ACC}_{\text {sin.masc }}$ & $\mathrm{DAT}+\mathrm{ACC}_{\text {sin.fem }}$ & $\mathrm{DAT}+\mathrm{ACC}_{\mathrm{pl} \text {.masc }}$ & $\mathrm{DAT}+\mathrm{ACC}_{\mathrm{pl} \text {.fem }}$ \\
\hline $1^{\mathrm{a} p}$ p.s. & mo $(\mathrm{me}+\mathrm{o})$ & $\mathrm{ma}(\mathrm{me}+\mathrm{a})$ & $\operatorname{mos}(\mathrm{me}+\mathrm{os})$ & $\operatorname{mas}(m e+a s)$ \\
\hline $2^{\mathrm{a}} \mathrm{p} . \mathrm{s}$ & to $(\mathrm{te}+\mathrm{o})$ & ta $($ te $+a)$ & tos $(\mathrm{te}+\mathrm{os})$ & tas (te+as) \\
\hline
\end{tabular}

${ }^{13}$ Se trata de procesos de naturaleza morfonológica y no fonológica debido a que la disimilación se produce en un contexto morfológico definido, es decir, ante la presencia de otros clíticos y no de cualquier segmento fónico. Así, es posible encontrar la secuencia fonológica le lo en español o ti ne en italiano en la que, por tanto, no se ha activado ninguna regla:

(i) Español

Compradle lodo

(ii) Italiano

Martina *te/ti negherà una spiegazione

Martina will deny an explanation

${ }^{14}$ En este artículo sólo trataremos las relaciones morfonológicas que se producen en el interior del grupo clítico y no las relaciones que pueden establecerse entre el grupo clítico y anfitrión. No obstante, en nuestra opinión tanto las relaciones entre clíticos como las del grupo clítico y su anfitrión son el resultado de los principios que regulan el proceso de inserción léxica que situamos en el interfaz sintáctico-fonológico (Camacho, 2002).

${ }^{15} \mathrm{Si}$ bien podemos considerar que tanto el mecanismo de fusión como de supresión pueden ser el resultado de que los rasgos morfológicos de dos elementos se fusionan en uno. 


\begin{tabular}{|c|c|c|c|c|}
\hline $3^{\text {a }}$ p.s. & lho $($ lhe + o) & lha $($ lhe $+a)$ & lhos (lhe+os) & lhas (lhe+as) \\
\hline $1^{\mathrm{a} p}$ p.p. & no-lo $($ nos $+[1] \mathrm{o})$ & no-la(nos + [1]a) & no-los (nos $+[1]$ os $)$ & no-las (nos $+[1]$ as) \\
\hline $2^{\mathrm{a} p}$ p.p & vo-lo $(\operatorname{vos}+[1] \mathrm{o})$ & vo-la (vos + [1]a) & vo-los (vos $+[1]$ os $)$ & vo-las (vos $+[\mathrm{I}]$ as) \\
\hline $3^{a}$ p.p & lho (lhes +o) & lha (lhes $+a)$ & lhos (lhes +os) & lhas (lhes +as) \\
\hline
\end{tabular}

No obstante, el análisis más pormenorizado de estos fenómenos es el realizado por Eulàlia Bonet sobre el catalán hablado en Barcelona. En este trabajo, Bonet (1995) se centra en la formación de los siguientes grupos clíticos [li], [↔lzi] y $[\leftrightarrow 1 \mathrm{l} \leftrightarrow \mathrm{ni}] .{ }^{16} \mathrm{De}$ acuerdo con sus explicaciones, la formación de la amalgama [1i], es el resultado de combinar las formas de tercera persona acusativo ho y el clítico ablativo en. No obstante, su forma fónica transparente (la esperada de acuerdo con el output de la sintaxis) hubiera correspondido a *ho en $\mathrm{o}$ *en ho:

(17) Catalán

Bonet (1995: 609)

Això, de l'armari, [li] trauré després

this from the closet [li] will-take-out $\left(1^{\text {st }}\right)$ later

I will take this out of the closet later

La amalgama [ $\leftrightarrow$ lzi] es el producto de combinar un clítico de tercera persona de acusativo els con la forma de dativo li. Esta forma contrasta con la agramaticalidad de las combinaciones *els li y*li'ls. Curiosamente, la forma opaca resultante contiene formas clíticas asociadas con formas con distintos valores morfosintácticos; por ejemplo, en (18), la forma opaca contiene la forma del plural, mientras que su referencia anafórica es singular:

(18) Catalán

Bonet (1995:610)

Els llibres, a en Quim, [↔lzi] donaré demà the books to the Quim [ $\leftrightarrow$ lzi] will-give $\left(1^{\text {st }}\right)$ tomorrow

I will give the books to Quim tomorrow

Por último, la forma [ $\leftrightarrow \mathrm{lz} \leftrightarrow \mathrm{ni}$ ], resultado de combinar la forma amalgamada [ $\leftrightarrow$ lzi] con el clítico de genitivo en, contrasta con las formas transparentes $*[\leftrightarrow l$ zin $]$ y $*[\mathrm{n} \leftrightarrow 1 \mathrm{li}]$. De $[\leftrightarrow \mathrm{lz}-\leftrightarrow \mathrm{n}-\mathrm{i}]$ podríamos decir que el clítico genitivo $[\leftrightarrow \mathrm{n}]$ se inserta en la forma compleja $[\leftrightarrow$ lzi]:

(19) Catalán

Bonet (1995:611)

De pomes, als nens [ $\leftrightarrow \mathrm{lz}-\leftrightarrow \mathrm{n}-\mathrm{i}]$ donaré demà

of apples to the children $[\leftrightarrow 1 \mathrm{z} \leftrightarrow \mathrm{ni}]$ will-give $\left(1^{\mathrm{st}}\right)$ tomorrow

I will give apples to the children tomorrow

Otro de los fenómenos característicos del español, italiano o serbocroata, es el resultado de la combinación de dos clíticos con características fonológicas similares. Estas formas

\footnotetext{
${ }^{16}$ Las amalgamas están representadas fonéticamente debido a que se trata de una variedad hablada al margen de la norma escrita.
} 
activan un proceso de disimilación por el que la forma del primer clítico del par cambia. Más aún, este cambio implica que el clítico es sustituido por otro miembro del paradigma (Bonet 1995:612; Grimshaw 1999, 2001). En español, la presencia de dos clíticos de tercera persona hace que el primer miembro del grupo se transforme o cambie por la forma se (se trata del llamado se espúreo).

$$
\begin{aligned}
& \text { (20) Español } \\
& \text { Se lo doy }
\end{aligned}
$$

En italiano, por el contrario, la combinación de los clíticos de dativo tercera persona singular masculino gli y femenino le con las formas de acusativo lo, la, li, le y ne, da como resultado las siguientes formas:

\begin{tabular}{|c|c|c|}
\hline СомB & CIÓN & AMALGAMA \\
\hline $\begin{array}{l}\text { gli } \\
\text { le }\end{array}$ & $\begin{array}{l}\text { Lo } \\
\text { la } \\
\text { li } \\
\text { le } \\
\text { ne }\end{array}$ & $\begin{array}{l}\text { glielo } \\
\text { gliela } \\
\text { glieli } \\
\text { gliele } \\
\text { gliene }\end{array}$ \\
\hline
\end{tabular}

$$
\text { (21) Italiano }
$$

Por su parte, en serbocroata, si el clítico de tercera persona singular femenino de acusativo je está seguido por el clítico de tercera persona auxiliar je, el primer miembro de la combinación es sustituido por el alomorfo $j u$ :

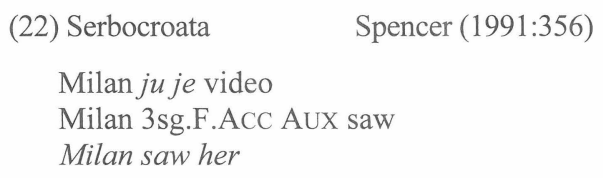

No obstante, si el grupo clítico está formado por el clítico genitivo je y el clítico auxiliar je, la secuencia no activa la regla de alomorfía sino que uno de los clíticos es elidido (Franks y King 2000:23).

Asimismo, en italiano, la combinación del clítico reflexivo si con el clítico impersonal $s i$, provoca que el primer si se convierta en $c i$ :

(23) Italiano

$\mathrm{Ci}_{\text {Ref. }} \mathrm{si}_{\text {Imp. lava }}$

Uno se lava (a sí mismo)

Hay que tener en cuenta que la tesis de la disimilación fonológica no puede explicar ejemplos como los de (24) y (25) en los que la secuencia compuesta por un se condicional seguido de un se reflexivo está permitida. Este es el caso del italiano o en el catalán de 
Valencia en donde dos clíticos de tercera persona de dativo y acusativo homófonos aparecen en un mismo grupo clítico: ${ }^{17}$

$$
\begin{aligned}
& \text { (24) Italiano Guasti y Nespor (1999) } \\
& \mathrm{Se}_{\mathrm{Cond}} \cdot \mathrm{Se}_{\mathrm{Ref} .} \text { la }_{\mathrm{ACU}} \text { sente } \\
& \text { If he feels like it }
\end{aligned}
$$

(25) Catalán hablado en Valencia Bonet (1995: 628)

Als xiquets, els llibres, els els portaré deprés

To the boys the books $3^{\text {rd }} \mathrm{pl} \mathrm{DAT} 3^{\text {rd }} \mathrm{pl} \mathrm{ACC}$ will take later

I will take the books to the boys later

Por último, en cuanto al proceso de supresión de uno de los clíticos de la secuencia, éste se produce en español y serbocroata si se combinan dos con la misma forma fónica:

(26) Español Perlmuttter (1970:202)

a) * Los honores $s_{\mathrm{j}} \operatorname{se}_{\mathrm{i}} \operatorname{los}_{\mathrm{j}}$ da a los generales

b) Los honores se los da a los generales

En síntesis, las combinaciones no permitidas de clíticos pueden variar de un sistema lingüístico a otro, si bien cada sistema lingüístico adopta una solución de entre un conjunto muy limitado de posibilidades. Tres son las soluciones que hemos podido identificar: (1) en español y serbocroata, en el supuesto de que el primer elemento sea un clítico genitivo, se suprime uno de los clíticos, (2) en catalán uno de los clíticos es realizado como pronombre tónico y (3) en italiano y serbocroata (cuando el primer miembro del grupo es un clítico de acusativo) se producen fenómenos de alomorfía.

En este apartado hemos comprobado que en la formación de los grupos clíticos intervienen tanto cuestiones de orden lineal (los clíticos son ordenados de acuerdo con criterios morfosintácticos relacionados con el caso y la persona gramatical) como fenómenos morfonológicos de fusión y amalgama. De nuevo, los datos parecen indicar que estamos tratando con un fenómeno morfológico. Por otro lado, esto no implica que ambos fenómenos deban ser explicados mediante los mismos mecanismos. De hecho, para examinar con detalle los problemas que plantean los grupos clíticos a la teoría de la gramática es necesario analizarlos por separado.

En cuanto al orden fijo que deben seguir los clíticos, la cuestión que debemos plantearnos es qué componente es el encargado de regular dicho orden y cuáles son los mecanismos que intervienen en el proceso. La elección del componente va a depender del modelo gramatical en el que desarrollemos el análisis. Así, por ejemplo, el modelo de gramática generativo clásico o la Teoría Antisimétrica de la Sintaxis de Kayne (1994) sostienen que el orden lineal de los elementos terminales sintácticos es el reflejo de la

\footnotetext{
17 Éste no es el único caso en que la conducta del si impersonal italiano difiere del de otras lenguas romances. Generalmente, el clítico impersonal y reflexivo ocupan la posición más a la izquierda del grupo clítico. Con todo, el clítico impersonal italiano puede ocupar la segunda posición del grupo clítico:
}

(i) Italiano

Lo si dice

Se dice (eso) 
estructura constitutiva sintáctica. Por tanto, los partidarios de esta hipótesis sostienen que el orden de los clíticos es el reflejo del orden jerárquico de las proyecciones funcionales en las que se alojan, bien sea debido a que han sido generados in situ, o bien se trate del lugar de aterrizaje del clítico tras una operación de movimiento (vid. Kayne 1975, 1994; Emonds 1975; Rudin 1996; Progovac 1993a, 1993b, 1996, Dimitrova-Vulchanova 1993, 1999; Terzi 1994, 1996a, 1996b, 1999, Franco 1991, 1993, entre otros).

En cambio, en las propuestas lexicalistas de Halpern (1995) y las hipótesis postlexicalistas de Bonet $(1991,1995)$ y Harris (1995), los grupos clíticos se forman en el componente morfológico. En los análisis que postulan la existencia de un componente postsintáctico en la arquitectura de la gramática, se entiende que la formación de grupos clíticos es un ejemplo de desajuste entre estructura sintáctica y fonológica, es decir, el output de la sintaxis no coincide con el input de la fonología. Así, el orden estricto que se respeta en el grupo clítico es el resultado de la aplicación de ciertos principios morfológicos que actúan como filtros del output de las configuraciones sintácticas, ya sea mediante la aplicación de una plantilla morfológica (vid. Perlmutter 1971;Hauge 1976; Ewen 1979, entre otros), o mediante la competición de los clíticos por la primera posición.

En cuanto a las formas opacas, los análisis sintácticos no tratan este tema. Los análisis morfológicos, por su parte, mantienen dos posturas, a saber, los análisis lexicalistas como el de Halpern (1995) sostienen que estos fenómenos son de tipo léxico y que, por tanto, deben ser tratados por los principios de la Fonología y Morfología Léxica, al tiempo que los análisis postlexicalistas de la Morfología Distribuida mantienen que estas formas se producen en el interfaz sintáctico-fonológico.

Nuestro objetivo consiste en determinar, desde el punto de vista descriptivo y explicativo, cuál de ellos es el más adecuado en un modelo de gramática modular y jerárquica en la que la relación entre componentes está controlada por los principios que regulan los interfaces.

\section{LA FORMACIÓN DE GRUPOS CLIITICOS EN EL COMPONENTE POSTSINTÁCTICO.}

Como ya hemos indicado, en este artículo intentamos demostrar que los análisis que dan cuenta de la formación de los grupos clíticos desde el componente sintáctico son inadecuados desde el punto de vista empírico. El orden interno de los grupos clíticos en los sistemas lingüísticos eslavos depende fundamentalmente del caso que representan (y no su función sintáctica), mientras que los grupos clíticos románicos dependen tanto de la persona gramatical como del caso que representa cada clítico. Es decir, el comportamiento de los clíticos pronominales eslavos y románicos parece estar sujeto a restricciones de tipo morfológico. Asimismo, los fenómenos morfonológicos que se observan en el interior del grupo clítico, a saber, los casos de fusión, alomorfía o elisión, parecen confirmar esta hipótesis.

La teoría lingüística nos ofrece la posibilidad de que los grupos clíticos puedan formarse en el componente morfológico léxico. Sin embargo, rechazamos dicha posibilidad puesto que sólo podría dar cuenta de la formación de los grupos clíticos categoriales. Si los clíticos de segunda posición tienen en la sintaxis el estatuto de categorías $\mathrm{D}^{\text {máx/mín }}$, sería necesario asumir que estos grupos clíticos se generan en el componente morfológico postsintáctico. Como acabamos de ver, los fenómenos morfonológicos que se observan en estos grupos son de la misma naturaleza que advertimos en los grupos formados por clíticos categoriales. 
Por tanto, concluimos que sería conveniente que la formación de los grupos clíticos se produzca, tanto en el caso de los clíticos categoriales como de segunda posición, mediante los mismos mecanismos. La solución que proponemos consiste en que los grupos clíticos de ambas clases se generen en el interfaz sintáctico-fonológico.

Esta hipótesis parece confirmarse con las construcciones de subida de clíticos:

(26) Serbocroata

(Franks y King 2000:244)

Marija ju je pustila da pliva

Marija it/her.Acc aux.3sg let C swims

Marija let it/her swim

En (26), observamos que dos clíticos subcategorizados por verbos diferentes pueden formar parte de un mismo grupo clítico. E incluso, pueden formar amalgamas entre ellos. Lo interesante de este fenómeno consiste en que éste se observa tanto en los sistemas lingüísticos eslavos como en los románicos. Si tenemos en cuenta que, en la sintaxis, los clíticos de estos sistemas lingüísticos poseen estatutos diferentes, la única manera de explicar por qué están sujetos a las mismas restricciones es suponer que éstos no están controlados por las reglas sintácticas.

El único análisis, del que tenemos noticias, que es compatible con este planteamiento que acabamos de hacer es la propuesta por Bonet (1991). En este modelo, las características idiosincrásicas de los grupos clíticos son consecuencia de ciertos desajustes producidos entre la estructura sintáctica y fonológica de estas secuencias de clíticos. El análisis de Bonet (1995:612) parte de la observación de que siempre y cuando se produzcan formas opacas en un sistema lingüístico dado, dichas formas son el resultado de la sustitución de uno de los elementos del grupo clítico por otro miembro de su paradigma; es decir, en ningún caso la forma opaca consiste en una secuencia fonológica arbitraria. Por ejemplo, el fenómeno del se espúreo del español residiría en la sustitución del clítico DAT le por la forma se. Lo interesante de este cambio radica en que, desde el punto de vista de la interpretación de estas construcciones, éste sólo afecta a la forma fónica del clítico, y no a los rasgos morfosintácticos interpretables de los que está compuesto. Por tanto, el mecanismo que da cuenta de la formación de los grupos clíticos debe estar localizado en un punto de la derivación entre el componente sintáctico y el fonológico. Del mismo modo, este mecanismo debe bloquear la posible derivación de secuencias fonológicas arbitrarias.

Durante la derivación, las reglas de empobrecimiento morfológico traducen las estructuras morfosintácticas de rasgos que componen los clíticos en información morfológica. Dicha información será emparejada, posteriormente, en el proceso de inserción léxica con información fonológica. Este proceso estará guiado por las reglas de inserción léxica las cuales relacionan los segmentos fonológicos con información sobre dónde será insertado dicho segmento. Por su parte, las piezas del Vocabulario se caracterizan por estar subespecificadas. Esto permite que las expresiones fonológicas sean emparejadas con posiciones sintácticas con las que no comparten cada uno de sus rasgos. En este sentido, en el proceso de inserción léxica, las piezas del Vocabulario compiten entre sí por alcanzar la misma posición. Este proceso está controlado por el Elsewhere Principle que garantiza que serán insertadas en primer lugar aquellas posiciones mejor especificadas. 
Con todo, no estamos de acuerdo con la hipótesis de la plantilla que Bonet (1995) nos ofrece para dar cuenta del orden interno de los grupos clíticos. En su lugar, proponemos que el proceso de linealización de los elementos terminales de la estructura sintáctica debe realizarse en el componente morfológico mediante la aplicación de ciertos principios de linealización del modelo de la Teoría de la Optimidad.

Uno de los pocos trabajos que desarrolla un análisis completo sobre el orden interno de los grupos clíticos románicos en el modelo de la Teoría de la Optimidad es el de Gerlach (1998). El análisis de Gerlach considera que el orden estricto de los grupos clíticos es consecuencia de que en el proceso de inserción léxica (interpretado como un proceso de input/output regulado por los principios de la Teoría de la Optimidad) el léxico no contiene candidatos que se adecuen fielmente a la especificación del input. Como consecuencia, la gramática debe escoger como alternativa el más óptimo de entre los candidatos que ofrece el Generador.

A diferencia de la hipótesis que defendemos en este trabajo, el análisis de esta autora asume que la formación de los grupos clíticos es el resultado de aplicar simultáneamente un conjunto de principios de naturaleza sintáctica, morfológica (y fonológica para el caso de los grupos clíticos opacos). Sin embargo, como veremos, su propuesta puede ser fácilmente adaptada a nuestros propósitos. En este sentido, para explicar cómo se ordena secuencialmente un grupo clítico y cómo se rechazan las combinaciones de clíticos no permitidas, Gerlach hace uso de los principios de alineamiento (los cuales tienen en cuenta la persona y la función sintáctica que realiza cada clítico) y la familia de restricciones de fidelidad MAX aplicados a funciones sintácticas (que impiden que ningún elemento sea elidido). En nuestra opinión, estas restricciones sintácticas pueden ser consideradas de naturaleza morfológica si, en lugar de hacer referencia a la función sintáctica de cada clítico, se tiene en cuenta su caso morfológico.

Debemos tener en cuenta que Gerlach adopta las propuestas de la subespecificación léxica de la Morfología Minimalista de Wunderlich y Fabri (1995). Esto implica que las entradas léxicas de los clíticos deben incluir información sobre la categoría a la que pertenecen. Esta autora considera que los clíticos tienen el estatuto de determinantes, en nuestro caso, proponemos que el estatuto puede variar dependiendo del sistema lingüístico a $\mathrm{D}^{\min / \operatorname{máx}}$ o Conc ${ }^{\text {mín/máx }}$. Asimismo deben especificar sus rasgos de concordancia y caso y, por último, deben explicitar que son elementos subcategorizados por el verbo. De esto se sigue que, en la sintaxis, el clítico se comporta como un argumento verbal y, por tanto, debe saturar la valencia de éste. ${ }^{18} \mathrm{La}$ entrada léxica que propone es la siguiente:

(27) Entrada léxica: $[+D]$; agr, case / +V.

Asimismo, cada una de las entradas léxicas de los clíticos incluye información sobre la persona gramatical, $[+1],[+2]$, el número $[+\mathrm{pl}]$, género $[+\mathrm{f}]$ y caso $[+\mathrm{hr}],[+\mathrm{lr}]$. Por tanto, el léxico del italiano incluiría la siguiente información:

${ }^{18}$ Esta entrada léxica no especifica a qué unidad lingüística corresponde el clítico pronominal: $\mathrm{D}^{\circ}$ o FD pleno. Además, no define al clítico como tal sino que la información en (30) puede corresponder perfectamente a un elemento pronominal tónico. Asimismo, no hace referencia a las características fonológicas del mismo, el ser fonológicamente defectivo, si bien esta información aparece en la secuencia de entrada que evalúa las restricciones que impone la sintaxis. Para evaluar la secuencia de clíticos en la sintaxis, el input debe contener información léxica del verbo y de los clíticos involucrados. 
(28) Léxico del italiano Gerlach (1998:11)

$$
\begin{array}{ll}
M i+1,+\mathrm{hr} & c i+1,+\mathrm{pl},+\mathrm{hr} \\
T i+2,+\mathrm{hr} & v i+2,+\mathrm{pl},+\mathrm{hr} \\
\text { Lo }+\mathrm{hr},-\mathrm{lr} & l i+\mathrm{pl},+\mathrm{hr},-\mathrm{lr} \\
\text { La }+\mathrm{f},+\mathrm{hr},-\mathrm{lr} & l e+\mathrm{f},+\mathrm{pl},+\mathrm{hr},-\mathrm{rr} \\
\text { Gli }+\mathrm{hr},+\mathrm{lr} & g l i+\mathrm{pl},+\mathrm{hr},+\mathrm{lr} \\
\text { Le +f, +hr, }+\mathrm{lr} &
\end{array}
$$

En (28) observamos que los clíticos de primera y segunda persona pueden funcionar indistintamente como clíticos de dativo o acusativo. Por tanto sólo están especificados para [+hr]. En cambio, en el caso de los clíticos de tercera persona es necesario diferenciar entre clíticos de acusativo y dativo, por lo que serán caracterizados como $[+\mathrm{hr},-\mathrm{lr}]$ y $[+\mathrm{hr},+\mathrm{lr}]$, respectivamente. Además, podemos comprobar que sólo son especificados los rasgos marcados que identifican las formas que serán seleccionadas prioritariamente del paradigma, mientras que los rasgos con valor [-] son añadidos por defecto (la única excepción son los clíticos de tercera persona de dativo). Esto indica que se hace uso del principio de subespecificación léxica aplicado al interfaz sintáctico-léxico. Recordemos que la teoría de la subespecificación léxica fue desarrollada por Bonet (1991) en el modelo de la Morfología Distribuida. Por tanto, la gramática exigirá en una posición determinada la presencia de una pieza léxica que sea compatible con los rasgos morfosintácticos asociados a ella en la medida de lo posible, pero que en ningún caso exige que coincidan exactamente.

Para Gerlach (1998), las restricciones sintácticas que garantizan la presencia de los clíticos son las siguientes (hemos simplificado la información para facilitar la exposición de nuestros argumentos):

(29) Restricción sintáctica: clitic linking is preferred

$$
\begin{array}{ll}
\operatorname{MAX}\left(\mathrm{C}_{\mathrm{O}}\right) & \text { Indirect object positions correspond with clitics. } \\
\operatorname{MAX}\left(\mathrm{C}_{\mathrm{DO}}\right) & \text { Direct object positions correspond with clitics } \\
\operatorname{DEP}\left(\mathrm{C}_{\mathrm{O}}\right) & \text { Object clitics correspond with object positions } \\
\text { IDENT(case) } & \text { Case features of corresponding elements are identical. } \\
\text { IDENT(agr) } & \text { Agreement features of corresponding elements are identical. }
\end{array}
$$

Con estos principios Gerlach (1998a) se asegura de que los clíticos tengan el mismo estatuto en la sintaxis que las FFDD con función de objeto, tal y como hemos visto en su entrada léxica, y que sólo aparezcan en construcciones en las que el verbo exija su presencia como sus argumentos. Así, $\mathrm{MAX}\left(\mathrm{C}_{\mathrm{IO}}\right)$ y $\mathrm{MAX}\left(\mathrm{C}_{\mathrm{DO}}\right)$ garantizan que las posiciones que corresponden a los objetos directos e indirectos sean ocupadas por clíticos. La restricción $\operatorname{DEP}\left(\mathrm{C}_{\mathrm{O}}\right)$, por su parte, garantiza que los clíticos sólo aparezcan cuando existan posiciones adecuadas de objeto disponibles. Este principio estará situado en una de las posiciones más altas de la jerarquía por lo que difícilmente será transgredido. IDENT(case) e IDENT(agr) exigen que los elementos con los que se les relacionan compartirán los mismos rasgos de caso y concordancia.

Una vez garantizado que el verbo ha seleccionado correctamente el conjunto de clíticos adecuados, Gerlach (1998) propone que éstos formen una secuencia morfológica. Dicha secuencia se adjuntará posteriormente al verbo. 
Por el contrario, si asumimos el modelo de componente morfológico descrito en la Morfología Distribuida por Bonet (1991, 1995), al entrar la derivación en dicho componente la información sintáctica debe ser suprimida. Para ello, propondremos que una serie de reglas morfológicas traduzcan la información sintáctica relativa a la función que realizan los clíticos a información morfológica, por lo que las restricciones de (29) serían consideradas morfológicas:

(30) Restricciones morfológicas

$\begin{array}{ll}\text { MAX }\left(\mathrm{C}_{\mathrm{DAT}}\right) & \text { Posiciones de Objeto Indirecto corresponden a clíticos de DAT. } \\ \operatorname{MAX}\left(\mathrm{C}_{\mathrm{AcU}}\right) & \text { Posiciones de Objeto Indirecto corresponden a clíticos de DAT. } \\ \text { DeP }\left(\mathrm{C}_{\mathrm{O}}\right) & \text { Posiciones de Objeto corresponden a clíticos de objeto. } \\ \text { IDENT(Caso }) & \text { Los rasgos de caso de estos elementos son idénticos. } \\ \text { IdENT(Conc) } & \text { Los rasgos de concordancia de los elementos son idénticos. }\end{array}$

Gerlach (1998) define, además, un conjunto de restricciones morfológicas denominadas de alineamiento que, junto a las restricciones de (29), garantizan el orden correcto de las secuencias. Dichas restricciones exigen que los clíticos compitan para ocupar la posición más a la izquierda de la secuencia (Gerlach 1998:15):

(31) Restricciones morfológicas

$\begin{array}{ll}\text { ALGN-L }(+1, C S) & 1^{\text {st }} \text { person clitics are leftmost in the C[litic] S[equence]. } \\ \text { ALGN-L }(+2, C S) & 2^{\text {nd }} \text { person clitics are leftmost in CS. } \\ \text { ALGN-L }(+ \text { lr,CS }) & \text { Indirect object clitics are leftmost in the CS. }\end{array}$

Estas restricciones exigen que el elemento que se sitúe más a la izquierda del grupo clítico sea un clítico de primera persona ALGN-L (+1,CS), un clítico de segunda persona, AlgN-L $(+2, \mathrm{CS})$ o un clítico de tercera persona de dativo, AlgN-L $(+\mathrm{lr}, \mathrm{CS})$. La tesis de Gerlach consiste en que, para dar cuenta del orden de los clíticos, los criterios morfológicos deben estar colocados en un nivel más alto en la jerarquía que las restricciones sintácticas de (29) y que nosotros sustituimos por (30).

La combinación de ambas clases de constricciones garantizarán, por tanto, el orden especial y estricto que se observa en los grupos clíticos haciendo referencia a la persona o el caso que representan, al tiempo que podrán rechazar las combinaciones no posibles de los sistemas lingüísticos. Si bien Gerlach se centra fundamentalmente en el italiano, su propuesta puede ser aplicada a la formación de grupos clíticos eslavos.

Veamos cómo se aplican dichas restricciones para dar cuenta de la formación de un grupo clítico italiano compuesto por la forma de tercera persona dativo gli y la forma de acusativo lo. Téngase en cuenta que el orden de los elementos del output sintáctico que corresponde al input del generador no está especificado.

(32) Italiano INPUT: DAT, ACU

CLÍTICOS: gli [+hr, +1r], lo [+hr, - lr $]$

\begin{tabular}{|l|l|l|l|}
\hline & & ALGN-L $(+\mathrm{lr}, \mathrm{CS})$ & $\operatorname{MAX}\left(\mathrm{C}_{\mathrm{O}}\right)$ \\
\hline \hline a. & Ф glie lo & & \\
\cline { 1 - 3 } & & & \\
\hline
\end{tabular}




\begin{tabular}{|c|c|l|l|}
\hline b. & lo gli & $* !$ & \\
\hline c. & Gli & & $* !$ \\
\hline d. & Lo & & $* !$ \\
\hline \hline
\end{tabular}

En (32), la restricción sintáctica $\operatorname{MAX}\left(\mathrm{C}_{\mathrm{O}}\right)$ exige que en el output estén presentes los clíticos especificados en el input, mientras que ALGN-L(+lr,CS) requiere que el clítico situado más a la izquierda de la secuencia sea el de acusativo (caracterizado por el rasgo $[+1 r])$. Ambas restricciones se sitúan en el mismo nivel de la jerarquía por lo que el incumplimiento de una de ellas supone una trasgresión fatal, por tanto, (b), (c) y (d) son descartados y la secuencia más óptima es la de (a), es decir, glielo.

Estas restricciones, además, dan cuenta del hecho de que no están permitidas las secuencias $* 1^{\mathrm{a}}, 2^{\mathrm{a}} \mathrm{o} * 1^{\mathrm{a}} / 2^{\mathrm{a}}-3^{\mathrm{a}}$ DAт puesto que los dos elementos de la secuencia de clíticos compiten por la misma posición. Recuérdese que la solución más óptima consiste en que el argumento dativo se realice como clítico y el de acusativo como FD completa, es decir, aquella en la que uno de los clíticos es elidido.

(33) Español $I_{N P U T} * 2 \mathrm{ACC} 3 \mathrm{DAT}$

\begin{tabular}{|l|l|l|l|l|l|}
\hline & & ALIGNMENT & $\operatorname{SPEC}\left(\mathrm{C}_{\mathrm{per}}\right)$ & $\operatorname{MAX}\left(\mathrm{C}_{\mathrm{DAT}}\right)$ & $\operatorname{MAX}\left(\mathrm{C}_{\mathrm{ACU}}\right)$ \\
\hline \hline a. & Le te & $* !$ & & & \\
\hline b. & Le & & $* !$ & & $*$ \\
\hline c. & $\Phi \quad t e$ & & & $*$ & \\
\hline \hline
\end{tabular}

Para este caso, son necesarios las restricciones sintácticas de $\operatorname{SPEC}\left(\mathrm{C}_{\mathrm{per}}\right)$, que exigen que el output contenga clíticos que compartan los mismos rasgos de persona que aparecen en el input y las restricciones de $\operatorname{MAX}\left(\mathrm{C}_{\mathrm{DAT}}\right)$ y $\operatorname{MAX}\left(\mathrm{C}_{\mathrm{ACU}}\right)$ que requieren que estén presentes dos clíticos de dativo y acusativo. Asimismo, es tenido en cuenta el requisito morfológico de Align. (Si bien no especifica cuál de las estricciones de la familia de alineamiento interviene, suponemos que se trata de ALGN-L(+lr,CS), puesto que el español cumple el requisito de DAT ACU, ejemplificado anteriormente). ALIGN y $\operatorname{SPEC}\left(\mathrm{C}_{\mathrm{per}}\right)$ están situados más altos en el ranking que $\operatorname{MAX}\left(\mathrm{C}_{\mathrm{DAT}}\right)$ y $\operatorname{MAX}\left(\mathrm{C}_{\mathrm{ACU}}\right)$. En este contexto, el output seleccionado será (c), es decir, la forma de dativo de segunda persona te puesto que aunque incumple el principio $\operatorname{MAX}\left(\mathrm{C}_{\mathrm{DAT}}\right)$, al no estar presente le, (a) y (b) incumplen fatalmente $\operatorname{SPEC}\left(\mathrm{C}_{\text {per }}\right)$ (habida cuenta de que las formas de tercera persona no están especificadas con el rasgo de persona en el léxico) y ALIGN (puesto que el clítico de acusativo precedería al de dativo).

En resumen, estos análisis parecen demostrar que los requisitos morfológicos de alineación exigen la trasgresión de ciertos principios sintácticos hasta el punto de que el output carece de uno de los elementos del input. Gerlach (1998:23) sugiere la siguiente jerarquía de rasgos para dar cuenta del orden de los clíticos de los sistemas lingüísticos románicos: 
(34) Align-L (+1, CS), Align-L (+2, CS), Align-L (+lr1, CS) $>$ MAX $\left(\mathrm{C}_{\mathrm{O}}\right)$

Por otro lado, para dar cuenta de la existencia de combinaciones no posibles propone los siguientes principios:

$$
\begin{array}{ll}
* 1+2 & \text { Align-L (+1, CS), o Align-L (+2, CS) } \\
* 3+1 / 2 & \text { Align-L (+1r, CS) o Align-L }(+1 /+2, \text { CS })
\end{array}
$$

Como hemos visto anteriormente, el rumano y el francés permiten algunas de las combinaciones agramaticales en español e italiano. El francés admite secuencias de clíticos formadas por elementos de tercera persona de acusativo y dativo con el orden 3 ACC $3_{\mathrm{DAT}}$. En cambio, en rumano están permitidos los grupos clíticos de tercera y primera persona con clíticos de segunda persona singular, el orden admitido es $1 / 3_{\mathrm{DAT}}-2 \mathrm{~s}_{\mathrm{Acc}}$. Para dar cuenta de estos datos, Gerlach considera necesario establecer una jerarquía entre las restricciones morfológicas, de modo que ALIGN-L(+1r,CS), en francés, y ALIGN-L(+2,CS), en rumano, sean considerados de rango inferior al resto de las restricciones morfológicas.

En síntesis, la formación de los grupos clíticos es el resultado de la intervención de los principios de dos niveles de representación: el componente sintáctico, encargado de distribuir los clíticos en la cláusula, y el interfaz sintáctico-fonológico en el que se determina el orden lineal de las unidades morfosintácticas y se produce el proceso de inserción léxica consistente en la sustitución de los rasgos morfológicos provenientes de la derivación por rasgos fonológicos. Como consecuencia de este proceso se generan tanto las formas opacas internas del grupo clítico como los fenómenos morfonológicos que observamos entre el grupo clítico y su anfitrión. Para llevar a cabo este proceso, asumiremos el modelo sintáctico de Principios y Parámetros, mientras que en el interfaz sintáctico-fonológico intervienen principios de la Morfología Distribuida y la Teoría de la Optimidad.

\section{REFERENCIAS BIBLIOGRÁFICAS}

BONET, E., Morphology after syntax: pronominal clitics in Romance, Tesis doctoral, Cambridge, Mass, MIT, 1991. [Distribuida por MIT Working Papers in Linguistics].

BONET, E., 1995. "Feature structure of romance clitics". Natural Language and Linguistic Theory, 13,4 (1995), pp 607-647.

CAMACHO, V., La arquitectura de la gramática: los clíticos pronominales románicos, Universidad de Sevilla, 205.

CAMACHO, V., "Caracterización sintáctica y fonológica de los clíticos pronominales románicos y eslavos", Actas del V Congreso de Lingüistica General, Universidad de León, 2004.

COROMINAS, J., Diccionario crítico etimológico de la lengua castellana, Madrid, Gredos, 1995-57.

CUNHA, C. y CINTRA, L., Nova gramatica do português contemporáneo, Lisboa, Joao Sa da Costa, 1984. 
DE BRAY, R., Guide to the Slavonic languages, vol 3: West Slavonic, Ann Arbor, Slavica, 1980.

Dimitrova-VUlCHANOVA, M., "Clitics in Slavic", Working Papers in Linguistics 18(1993),pp.1-50.

DIMITROVA-VULCHANOVA, M. 1999. "Clitics in the Slavic languages", en H. Riemsdijk (ed.) Clitics in the languages of Europe, Berlín, Mouton de Gruyter, pp.83-122.

EMONDS, J. "A transformational analysis of French clitics without positive output constraints", Linguistic Analysis 1(1975), pp. 3-24.

EWEN, R., A grammar of Bulgarian clitics, Tesis doctoral de la Universidad de Washington, 1979.

FERNÁNDEZ SORIANO, O., "El pronombre personal átono. Formas y distribuciones. Pronombres átonos y tónicos", en Gramática descriptiva de la lengua española, dirs. I. Bosque y V. Demonte, Madrid: Espasa, 1999, pp.1209-1273.

FRANCO, J., "Spanish object clitics as verbal agreement", MIT Working Papers in Linguistics 14 (1991), pp. 99-114.

FRANCO, J., On object agreement in Spanish, Tesis doctoral, University of Southern California, 1993. Distribuida por GSIL.

FRANKS, S., "Position paper: clitics in Slavic", Workshop on Comparative Slavic morphosyntax, Spencer, Indiana, 1998.

FRANKS, S., "Clitics at the interface", en Clitic phenomena in European languages, eds. M. Den Dikken y F. Beukema, Amsterdam, John Benjamins., 2000, pp. 1-46

FRANKS, S. y.King, T.H., A handbook of Slavic clitics, Nueva York, Oxford University Press, 2000.

GERLACH, B., "Restrictions on clitic sequences and conditions on the occurrence of clitics in romance", en Theories des Lexikons, n. 105 (1998).

GRIMSHAW, J., "The alignment of Romance clitics," en Workshop on perspectives on clitic and agreement affix combinations, LSA Linguistic Institute, University of Illinois, Urbana-Champaign Campus, 1999.

GRIMSHAW, J., "Optimal clitic position and the Lexicon in Romance clitic system", en Optimality theoretic syntax, eds. G. Legendre, J. Grimshaw y S. Vikner. Cambridge, Mass, MIT Press, 2001. También en Rutgers Optimality Archive. Responsable: Alan Prince. Internet. 21/11/04. $<$ http://roa.rutgers.edu/view.php3?id=701 >

GUASTI, T. y NESPOR, M., 1999. "Is syntax phonology free?”, en Phrasal phonology, eds. R. Kager y W. Zonneveld, Dordrecht, Foris,1999, pp. 125-159.

HARRIS, J., "The morphology of Spanish clitics", en Evolution and revolution in linguistic theory, eds. H. Campos y P. Kempchinsky, Washington, Georgetown University Press, 1995, pp. 168-197.

KAYNE, R., French syntax: The transformational cycle, Cambridge, Mass, MIT Press, 1975. 
KAYNE, R., he antisymmetry of syntax. Cambridge, Mass, MIT Press, 1994.

LONGA, V., LORENZO, G., y RIGAU, G., "Subject clitics and clitic recycling: locative sentences in some Iberian Romance languages", Journal of Linguistics 34 (1998), pp.125-164.

MONACHESI, P., A lexical approach to Italian cliticization, Standford, CLSI Publications, 1999.

MUYSKEN, P., "Quechua word structure", en F.Heny (ed), Binding and filtering, Cambridge, Mass, MIT Press, 1981.

PERLMUTTER, D., "Surface structure constraints in syntax", Linguistic Inquiry 1.2 (1970), pp. 185-255.

PERLMUTTER, D., Deep and surface structure constraints in syntax, Nueva York, Holt, Rinehart and Winston, 1971. [Tesis doctoral, MIT, Cambridge, Mass.. 1968]

POPESCU, A., Objektklitika und Argumentlinking im Rumänischen. Tesis de máster, Heinrich-Heine-University, Düsseldorf, 1997.

PROGOVAC, Lj., "Locality and subjunctive-like complements in Serbo-croatian", Journal of Slavic Linguistics, 1 (1993a), pp.116-144.

PROGOVAC, Lj., "Subjunctive: The (Mis)behaviour of anaphora and negative polarity" The Linguistic Review, 10 (1993b), pp.37-59.

PROGOVAC, Lj., "Clitics in Serbian/Croatian deriving second position", en Approaching second: second position clitics and related phenomena, eds. A. Halpern y A. Zwicky, Stanford, CSLI Publications, 1996, pp. 411-428.

RIEMSDIJK, H., "Clitics: a state-of-the-art report", en Clitics in the languages of Europe, ed. H. Riemsdijk, Berlín, Mouton de Gruyter, 1999, 1-32.

RUDIN, C., 1996. On pronominal clitics. En Papers from the first conference on formal approaches to South Slavic Languages, eds. M. Dimitrova-Vulchanova y L Hellan, vol 28, University of Trodheim Working Papers in Linguistics, 1996, pp. 229-246.

SIMPSON, J., y WITHGOTT, M., 1986. "Pronominal clusters and templates", en The Syntax of pronominal clitics. Syntax and semantics, vol. 19, ed. H. Borer,. Orlando, Academic Press, 1986, 149-174.

SPENCER, A., Morphological theory, Oxford, Basil Blackwell, 1991.

SPORTICHE, D., "Clitic constructions", en Phrase structure and the lexicon, eds. J. Rooryck y L. Zaring, Dordrecht, Kluwer Academic Publishers, 1996, pp. 213-76.

TERZI, A., "Clitic climbing from finite clauses and long head movement", Catalan Working Papers in Linguistics 3.2(1994), pp. 97-122.

TERZI, A., "Clitic climbing from finite clauses and tense raising", Probus 8 (1996a):273295.

TERZI, A., 1996b, "The linear correspondence axiom and the adjunction sites of clitics", en Configurations: essays on structure and interpretation, ed. A. M. Di Sciullo, Somerville, Cascadilla Press, 1996b, pp. 185-199. 
TERZI, A., "Clitic combinations, their host and their ordering", Natural Language and Linguistic Theory 17 (1999), pp. 85-121.

VOS, R., y VESELOVSKÁ, L., 1999. Clitic questionaire. En Clitics in the languages of Europe, ed. H. Riemsdijk, Berlín, Mouton de Gruyter, 1999, pp. 891-1009.

WUNDERLICH, D., y FABRI, R., "Minimalist morphology: an approach to inflection", Zeitschrift für Sprachwissenschaft 14 (1995), pp. 236-294. 
